\title{
The Long-Term Progression Free Survival After the Combination of Radiotherapy with Concurrent Chemotherapy of Nimotuzumab And Temozolomide Followed Adjuvant Temozolomide In Recurrent Anaplastic Astrocytoma
}

\author{
Xueming Xia ${ }^{1}$, Feng Wang ${ }^{1}$, Wei Du ${ }^{1}$, Tao Ning ${ }^{1}$, Yong Luo ${ }^{1}$, XueLei Ma ${ }^{1}$, Lei Liu ${ }^{1}$ Hong Zhang $^{1}$ and Xiaoqi Xie ${ }^{2 *}$
}

${ }^{1}$ Department of Head and neck Oncology, West China Hospital, The People's Republic of China

${ }^{2}$ Department of Critical Care Medicine, West China Hospital, China

Submission: March 22, 2019; Published: April 12, 2019

*Corresponding author: Xiaoqi Xie, Department of Critical Care Medicine, West China Hospital, Sichuan University. The People's Republic of China

\begin{abstract}
The management of recurrent high-grade gliomas is highly challenging, and treatment outcome remains invariably poor. High-grade gliomas (HGGs) are highly malignant tumor, which complete surgical resection of all microscopic extensions cannot be always achieved. All high-grade gliomas nearly recur and survival following disease progression is doomed to be approximately 6 months for GBMs and 10 months for anaplastic gliomas. Therapy options for recurrent HGGs are limited and may include surgery, re-irradiation, chemotherapy and targeted therapy. We present a case of a 33-year-old male with recurrent anaplastic astrocytoma after initial surgery. The patient underwent the treatment of radiation therapy with concurrent chemotherapy of nimotuzumab and oral temozolomide for 6 weeks followed by six cycles of adjuvant temozolomide for tumor local recurrences, and the patient still do not relapse with a long-term progression free survival lasting seven years. The median survival in patients with recurrent anaplastic astrocytoma is usually 10 months after recurrence, and this unique case illustrates that comprehensive therapy can achieve long-term survival in select situations. We recommend that the treatment of each recurrent patient should be based on each clinical situation and aspire for quality of life and improved longevity.
\end{abstract}

Keywords: PFS; Temozolomide recurrent anaplastic astrocytoma

Abbreviations: HGGs: High-Grade Gliomas; GBMs: Glioblastomas; PFS: Progression Free Survival; TM: Temozolomide; mTTP: Median TimeTo-Tumor Progression; mOS: Median Overall Survival; MGMT: Methylguanine-DNA Methyltransferase; OS: Overall Survival; SRS: Stereotactic Radiosurgery; EGFR: Epidermal Growth Factor Receptor; RT: Radiotherapy

\section{Introduction}

Gliomas which originated from glial cells and made up 80\% of all primary CNS tumors in the United States were classified as "low grade" (WHO grades I and II) and "high grade" (WHO grades III and IV) according to histopathologic changes [1,2]. High-grade gliomas (HGGs) are related with poor prognosis and consist of anaplastic gliomas (anaplastic astrocytoma, anaplastic oligodendroglioma and anaplastic oligoastrocytoma; WHO grade III) and glioblastomas (GBMs; WHO grade IV). The current standard therapy for newly diagnosed GBMs involves maximal safe resection followed by radiotherapy (RT) with concomitant and adjuvant temozolomide (TMZ) [3-5]. Despite optimal therapy, the median time-to-tumor progression (mTTP) for patients with newly diagnosed GBMs is approximately 6.9 months and these tumors unavoidably relapse with a median overall survival (mOS) of only 15 months [3]. However, the treatment of anaplastic gliomas is abroad controversial topics in neuro-oncology. By above therapy, anaplastic gliomas achieves a mTTP of about 1.2 to 2.6 years and a mOS of 2 to 7 years $[4,6,7]$.

Compared with anaplastic oligodendroglioma, treatment of the anaplastic astrocytoma has been less optimistic. This tumor is more resistant to therapy and patients have a shorter mOS of only 2 to 3 years. In the United States, most oncologists treat anaplastic astrocytoma patients with maximal safe resection followed by field radiotherapy with concurrent and adjuvant chemotherapy (temozolomide), same to the regimen now deemed as the standard of care for GBMs [3]. Moreover, all HGGs nearly 


\section{Global Journal of Reproductive Medicine}

recur and survival following disease progression is doomed to be approximately 6 months for GBMs and 10 months for anaplastic gliomas [8,9]. Treatment options for recurrent HGGs include re-resection, chemotherapy, or re-irradiation. But, the optimal treatment strategies for recurrent HGGs are still unknown and the randomized control trials which contrast active intervention and determine standard therapy are absent. We herein report the case of a recurrent anaplastic astrocytoma patient with the long-term progression free survival (PFS) after the combination of radiation therapy with concurrent chemotherapy of nimotuzumab and temozolomide followed adjuvant temozolomide.

\section{Case Report}

We present the unique case of a 33-year-old gentleman who initially presented with a several days history of headaches behind the right brain, with associated "vomiting". There were no complaints of numbness, weakness, or any visual changes. Physical examination of the patient revealed that he had a depressed superficial reflex in the right limbs and positive pyramidal tract signs. A MRI scan at that time revealed a large, irregularly enhancing mass in the right frontal lobe that was associated with marked edema, bleeding and mass effect. These symptoms relieved with the treatment of dehydration to reduce intracranial pressure. Following the consultation with a neurosurgeon and discussing the probability of a brain tumor and therapeutic regimen, the patient experienced a right frontal lobe Lobectomy in local hospital in October 2008. The final pathology was anaplastic astrocytoma (WHO grade III).

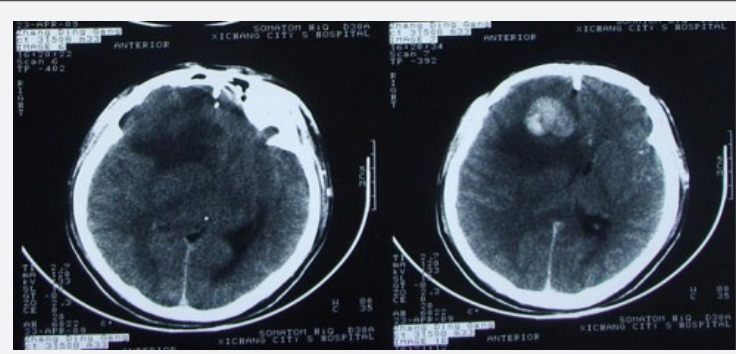

Figure 1: In April 2009, a follow up CT scan revealed local recurrence in the operative bed.

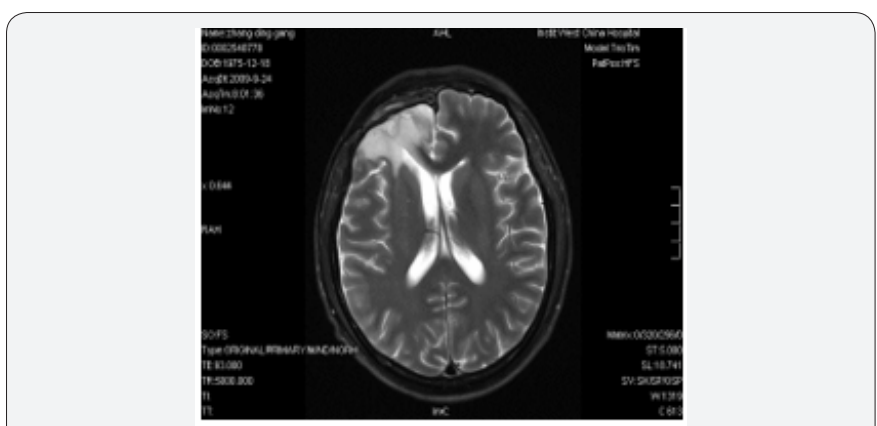

Figure 2: The follow up MRI scan in 3 months after radiotherapy

The patient recovered well from surgery, and his headaches were resolved. Adjuvant treatment options were explained to him and included radiotherapy plus concomitant and adjuvant
Temozolomide. But the patient refused further therapy. In April 2009, a follow-up CT scan revealed local recurrence in the operative bed (Figures 1-3). And the patient was treated in West China Hospital. The pathology department in West China Hospital held a pathological consultation with tumor section in local hospital and diagnosed anaplastic astrocytoma (WHO grade III) containing a few of oligodendroglioma ingredients with histopathologic changes of partial necrosis, mitotic activity and endothelial cell proliferation (Figure 4). KRAS mutation was detected in codon 12 and codon 13 and the DNA repair enzyme 06-methylguanineDNA methyltransferase (MGMT) promoter methylation was positive. Chromosomal $1 \mathrm{p}$ and $19 \mathrm{q}$ co-deletion was detected by FISH (Figures 5\&6). Isocitrate dehydrogenase 1 mutation (codon 132) was detected by PCR and Sanger sequencing and Isocitrate dehydrogenase 2 (codon 172) was wild type (Figures 7\&8).

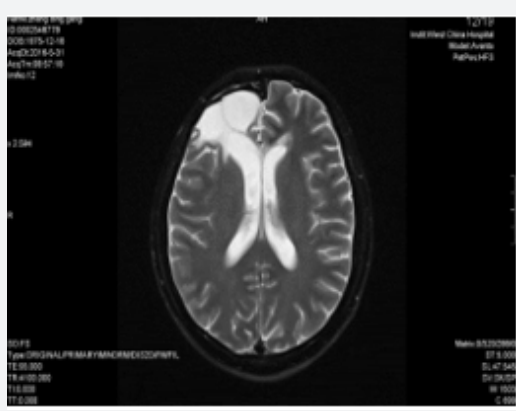

Figure 3: The latest MRI scan revealed no recurrence in May 31,2016 .

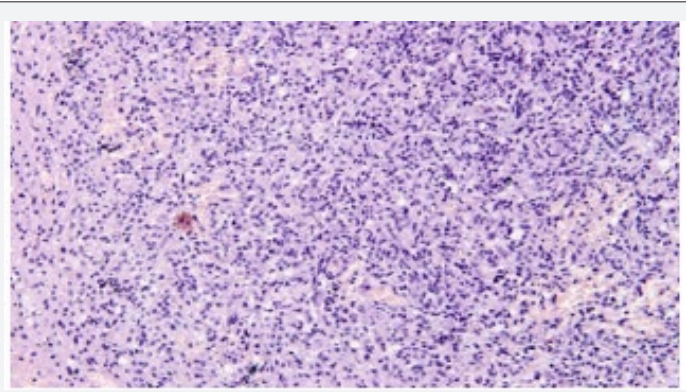

Figure 4: The patient's pathological image.

Starting on May 22, 2009, the patient underwent whole brain radiotherapy to a dose of $4000 \mathrm{cGy}$ and local lesion radiotherapy to a dose of 6000 cGy (fractionated focal irradiation in daily fractions of 2 Gy given 5 days per week for 6 weeks) with concurrent combined chemotherapy of intravenous nimotuzumab (100 mg every 1 weeks) and oral temozolomide (75 mg per square meter of body-surface area per day) for 6 weeks followed by six cycles of adjuvant temozolomide (150 mg per square meter for 5 days during each 28-day cycle). Treatment was generally well tolerated, and the adverse events were nausea, vomiting and hematologic toxicities, which all of them were classified as mild. Following his radiotherapy and chemotherapy, he was followed clinically as well as with surveillance MRI scans at regular intervals. The follow-up MRI scan in 3 months after radiotherapy were as follows (Figure 
2). The latest MRI scan revealed no recurrence in May 31, 2016 (Figure 3). Until now, the patient is still alive with a long-term progression free survival lasting seven years. And the survival following the initial diagnosis is up to eight years.

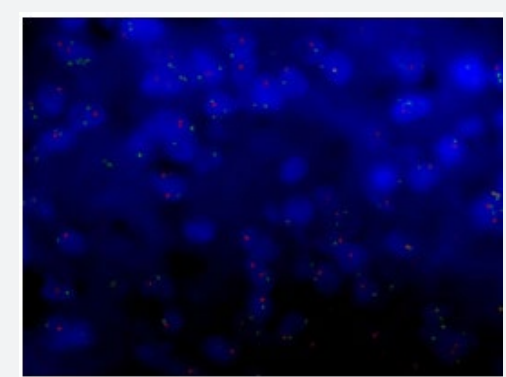

Figure 5: The patient's FISH image about chromosomal 1p/1q probe.

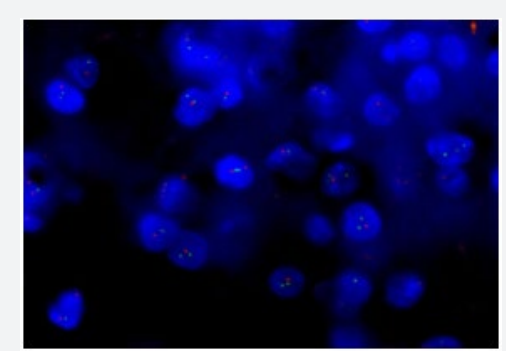

Figure 6: The patient's FISH image about chromosomal 19q/19p probe.

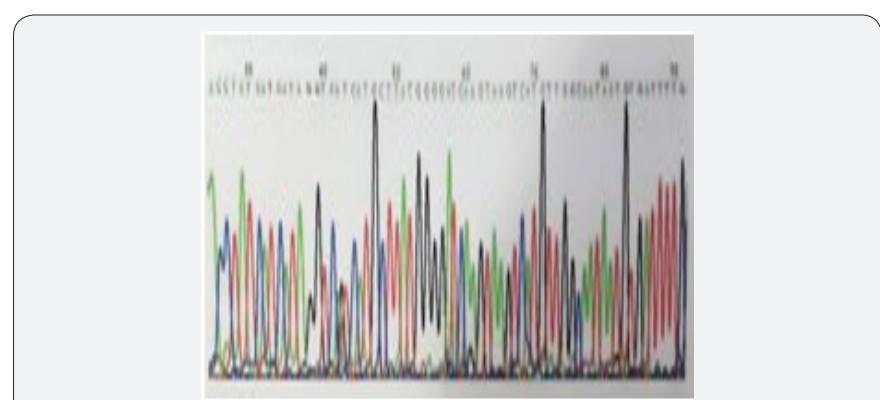

Figure 7: The patient's Isocitrate dehydrogenase 1.

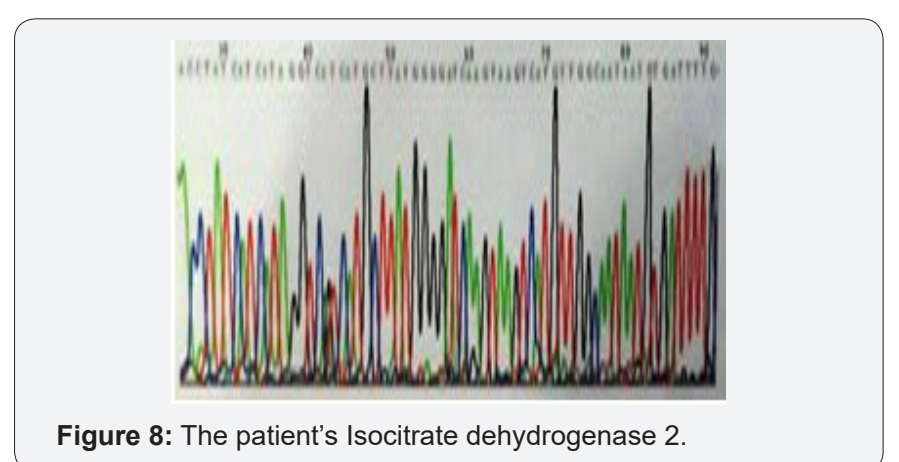

\section{Discussion}

A high rate of local recurrences has been observed after multi-disciplinary therapy in high-grade gliomas (HGGs) patients.10 Important variables predicting longer survival include oligodendroglial cell line, extent of resection, and younger age
( $<50$ years) [11]. Moreover, gliomas with the DNA repair enzyme 0-6-methylguanine-DNA methyltransferase (MGMT) promoter methylation, chromosomal $1 \mathrm{p}$ and $19 \mathrm{q}$ co-deletion or isocitrate dehydrogenase 1 and 2 (IDH1/2) mutations is associated with a better prognosis [12-15]. In this patient, the chromosomal $1 \mathrm{p}$ and $19 \mathrm{q}$ co-deletion was detected by FISH; and the MGMT promoter methylation was positive and Isocitrate dehydrogenase 1 was mutation type, which was one of the reason of long-term progression free survival (PFS). PFS is associated with overall survival (OS) and has become a marker for evaluating the efficacy of treatments in recurrent HGGs patients. The 6-month PFS (PFS6) for recurrent GBM ranges from 9 to 21\% [8,9,16], while PFS6 for anaplastic gliomas have ranged from 37 to $48 \%[17,18]$. In our report, the patient still does not progress with a long-term PFS lasting seven years. Although the prognosis of recurrence HGGs patients is invariably poor, it is vital just as this case to improve the quality of life and prolong survival by multimodal therapy.

Therapy options for recurrent HGGs are limited and may include surgery, re-irradiation, chemotherapy and targeted therapies. For recurrent HGGs patients which were subjected to clinical deterioration on account of mass effect, surgery might be one of option. But, until now, no prospective researches demonstrate that re-surgery can improve the survival for recurrent HGGs patients. Moreover, due to the application of targeted therapies, a fewer proportion of recurrent HGGs patients are going through diagnostic or therapeutic re-surgery.

For HGGs patients, primary therapy most involves adjuvant radiotherapy with the maximum tolerated dose. The dose limitation of healthy brain tissue and the short duration between initial radiation therapy and recurrence confined the use of reirradiation therapy for recurrent HGGs with conventional external beam radiotherapy, which may have an influence on patient's quality of life. However, Romanelli et al' s research that evaluated the efficacy of stereotactic radiosurgery (SRS) in recurrent glioma have indicated median OS from 7.5 to 30 months after treatment [19]. And in Mahajan's research [20], recurrent GBM patients treated with SRS achieved a longer median OS compared with untreated patients [20]. Hypofractionated stereotactic irradiation might be another choice for recurrent glioma. According to Fogh, et al. [21] research, the median OS after re-irradiation with a hypofractionated protocol (3.5 Gy per fraction up to a median dose of $35 \mathrm{~Gy}$ ) over a 2-week period was 11 months. In this case, because patient did not receive any further treatment after surgery, conventional external beam radiotherapy (whole brain radiotherapy to a dose of $4000 \mathrm{cGy}$ and local lesion radiotherapy to a dose of $6000 \mathrm{cGy}$ ) plus concomitant and adjuvant Temozolomide were recommended to him and the progression free survival after irradiation was seven years.

The efficacy of chemotherapy in recurrent HGGs was limited. And many chemotherapeutic agents, including single agents and combination chemotherapy agents, have been used to treat recurrent HGGs [22,23]. Temozolomide, PCV and bevacizumab are some of the treatment choice according to several researches [24-27]. 


\section{Global Journal of Reproductive Medicine}

Temozolomide was evaluated in recurrent HGGs before becoming the first line chemotherapy for glioma. The recurrent anaplastic glioma patients that were treated with single-agent TMZ showed a $35 \%$ response rate and PFS6 of 46\% [28]. Bevacizumab has been authorized by FDA as the treatment of recurrent GBMs [29]. Moreover, Nimotuzumab is a humanized monoclonal antibody which binds the extracellular domain of epidermal growth factor receptor (EGFR) and blocks EGFR signal pathway activation [30]. And the efficacy of nimotuzumab in HGGs is depended on the combination of nimotuzumab with radiochemotherapy or radiotherapy. Solomon et al. [31] research has illustrated the median survival time of the combination of nimotuzumab with radiotherapy was 12.4 months or 27.0 months for patients with GBM or anaplastic astrocytoma patients. In our case, the chemotherapy agents were the concurrent combined chemotherapy of intravenous nimotuzumab and oral temozolomide for 6 weeks followed by six cycles of adjuvant temozolomide. And the efficacy was surprising. Thus, nimotuzumab might be one of the chemotherapy choice and more randomized clinical trials need to be done to clarify the efficacy of nimotuzumab.

\section{Conclusion}

This is the first reported case to our knowledge of a patient with recurrent anaplastic astrocytoma who achieved PFS lasting seven years following treatment with the combination of radiation therapy with temozolomide and nimotuzumab. The patient tolerated the therapy well and do not relapse again until now. This case highlights the unique biology of individual glioma and that the combination of radiation with chemotherapy and targeted therapy in the recurrent setting can have a significantly influence on overall survival for specific patients. We will continue doing research in this area, especially in the molecular characteristics of individual tumors. Individualized treatment according to each unique patient's situation will improve survival as well as quality of life.

\section{References}

1. Ostrom QT, Gittleman H, Liao P, Rouse C, Chen Y, et al. (2014) CBTRUS statistical report: primary brain and central nervous system tumors diagnosed in the United States in 2007-2011. Neuro-oncology 4: iv163.

2. Louis DN, Ohgaki H, Wiestler OD, Cavenee WK, Burger PC, et al. (2007) The 2007 WHO classification of tumours of the central nervous system. Acta neuropathologica 114(2): 97-109.

3. Stupp R, Mason WP, van den Bent MJ, Weller M, Fisher B, et al. (2005) Radiotherapy plus concomitant and adjuvant temozolomide for glioblastoma. N Engl J Med 352(10): 987-996.

4. Wen PY, Kesari S (2008) Malignant gliomas in adults. N Engl J Med 359(5): 492-507.

5. Stupp R, Hegi ME, Mason WP, van den Bent MJ, Taphoorn MJ, et al. (2009) Effects of radiotherapy with concomitant and adjuvant temozolomide versus radiotherapy alone on survival in glioblastoma in a randomised phase III study: 5-year analysis of the EORTC-NCIC trial. Lancet Oncol 10(5): 459-466.

6. Intergroup Radiation Therapy Oncology Group T, Cairncross G, Berkey B, Shaw E, Jenkins R, et al. (2006) Phase III trial of chemotherapy plus radiotherapy compared with radiotherapy alone for pure and mixed anaplastic oligodendroglioma: Intergroup Radiation Therapy Oncology Group Trial 9402. J Clin Oncol 24(18): 2707-2714.

7. Van den Bent MJ, Carpentier AF, Brandes AA, Sanson M, Taphoorn MJ, et al. (2006) Adjuvant procarbazine, lomustine, and vincristine improves progression-free survival but not overall survival in newly diagnosed anaplastic oligodendrogliomas and oligoastrocytomas: a randomized European Organisation for Research and Treatment of Cancer phase III trial. J Clin Oncol 24(18): 2715-2722.

8. Lamborn KR, Yung WK, Chang SM, Wen PY, Cloughesy TF, et al. (2008) Progression-free survival: an important end point in evaluating therapy for recurrent high-grade gliomas. Neuro Oncol 10(2): 162-170.

9. Wong ET, Hess KR, Gleason MJ, Jaeckle KA, Kyritsis AP, et al. (1999) Outcomes and prognostic factors in recurrent glioma patients enrolled onto phase II clinical trials. J Clin Oncol 17(8): 2572-2578.

10. Niyazi M, Schnell O, Suchorska B, Schwarz SB, Ganswindt U, et al. (2012) FET-PET assessed recurrence pattern after radio-chemotherapy in newly diagnosed patients with glioblastoma is influenced by MGMT methylation status. Radiother Oncol 104(1): 78-82.

11. Wick W, Hartmann C, Engel C, Stoffels M, Felsberg J, et al. (2009) NOA-04 randomized phase III trial of sequential radiochemotherapy of anaplastic glioma with procarbazine, lomustine, and vincristine or temozolomide. J Clin Oncol 27(35): 5874-5880.

12. Cairncross G, Wang M, Shaw E, Jenkins R, Brachman D, et al. Phase III trial of chemoradiotherapy for anaplastic oligodendroglioma: longterm results of RTOG 9402 J Clin Oncol 31(3): 337-343.

13. Hegi ME, Diserens AC, Gorlia T, Hamou MF, de Tribolet N, et al. (2005) MGMT gene silencing and benefit from temozolomide in glioblastoma. N Engl J Med 352(10): 997-1003.

14. Sanson M, Marie Y, Paris S, Idbaih A, Laffaire J, et al. (2009) Isocitrate dehydrogenase 1 codon 132 mutation is an important prognostic biomarker in gliomas. J Clin Oncol 27(25): 4150-4154.

15. Wick W, Platten M, Meisner C, Felsberg J, Tabatabai G, et al. (2012) Temozolomide chemotherapy alone versus radiotherapy alone for malignant astrocytoma in the elderly: the NOA-08 randomised, phase 3 trial. Lancet Oncol 13(7): 707-715.

16. Yung WK, Albright RE, Olson J, Fredericks R, Fink K, et al. (2000) A phase II study of temozolomide vs. procarbazine in patients with glioblastoma multiforme at first relapse. Br J Cancer 83(5): 588-593.

17. Levin VA, Ictech S, Hess KR (2007) Impact of phase II trials with progression-free survival as end-points on survival-based phase III studies in patients with anaplastic gliomas. BMC Cancer 7(1): 106-106.

18. Walbert T, Gilbert MR, Groves MD, et al. (2011) Combination of 6-thioguanine, capecitabine, and celecoxib with temozolomide or lomustine for recurrent high-grade glioma. J Neurooncol 102(2): 273280.

19. Romanelli P, Conti A, Pontoriero A, Ricciardi GK, Tomasello F, et al. (2009) Role of stereotactic radiosurgery and fractionated stereotactic radiotherapy for the treatment of recurrent glioblastoma multiforme. Neurosurgical focus 27(6): E8.

20. Mahajan A, McCutcheon IE, Suki D, et al. (2005) Case-control study of stereotactic radiosurgery for recurrent glioblastoma multiforme. J Neurosurg 103(2): 210-217.

21. Fogh SE, Andrews DW, Glass J, Curran W, Glass C, et al. (2010) Hypofractionated stereotactic radiation therapy: an effective therapy for recurrent high-grade gliomas. J Clin Oncol 28(18): 3048-3053.

22. Quant EC, Drappatz J, Wen PY, Norden AD (2010) Recurrent high-grade glioma. Curr Treat Options Neurol 12(4): 321-333.

23. Schmidt F, Fischer J, Herrlinger U, Dietz K, Dichgans J, et al. (2006) PCV chemotherapy for recurrent glioblastoma. Neurology 66(4):587-589. 
24. Chamberlain MC (2002) Salvage chemotherapy with CPT-11 for recurrent glioblastoma multiforme. J Neurooncol 100(2): 1213-1220.

25. Minniti G, Muni R, Lanzetta G, Marchetti P, Enrici RM (2009) Chemotherapy for glioblastoma: current treatment and future perspectives for cytotoxic and targeted agents. Anticancer Res 29(12) 5171-5184.

26. Stupp R, Hegi ME, van den Bent MJ, Mason WP, Weller M, et al. (2006) Changing paradigms--an update on the multidisciplinary management of malignant glioma. Oncologist 11(2): 696-706.

27. Trent S, Kong A, Short SC, Traish D, Ashley S, et al. (2002) Temozolomide as second-line chemotherapy for relapsed gliomas. J Neurooncol 57(3): 247-251.

28. Yung WK, Prados MD, Yaya-Tur R, Rosenfeld SS, Brada M, et al. Multicenter Phase II Trial of Temozolomide in Patients with Anaplastic
Astrocytoma or Anaplastic Oligoastrocytoma at First Relapse. J Clin Oncol 17(9): 2762-2771.

29. Friedman HS, Prados MD, Wen PY, et al. (2009) Bevacizumab alone and in combination with irinotecan in recurrent glioblastoma. J Clin Oncol 27(28): 4733-4740

30. Mateo C, Moreno E, Amour K, Lombardero J, Harris W, et al. (1197) Humanization of a mouse monoclonal antibody that blocks the epidermal growth factor receptor: recovery of antagonistic activity. Immunotechnology 3(1):71-81.

31. Solomon MT, Miranda N, Jorrín E, Chon I, Marinello JJ, et al. (2014) Nimotuzumab in combination with radiotherapy in high grade glioma patients: a single institution experience. Cancer Biol Ther 15(5): 504 509.

Your next submission with Juniper Publishers
will reach you the below assets
- Quality Editorial service
- Swift Peer Review
- Reprints availability
- E-prints Service
- Manuscript Podcast for convenient understanding
- Global attainment for your research
- Manuscript accessibility in different formats
( Pdf, E-pub, Full Text, Audio)
- Unceasing customer service
Track the below URL for one-step submission
https://juniperpublishers.com/online-submission.php

